\section{Images des récepteurs des messagers chimiques et des médicaments dans le cerveau}

Le système nerveux central est désormais accessible à l'exploration par des méthodes d'imagerie dynamiques. Celles-ci permettent de faciliter le diagnostic, de suivre le métabolisme des médicaments, et de mieux comprendre l'étiologie des maladies mentales.

\section{William Rostène \\ Rémi Quirion \\ Alain Beaudet Bernard Mazière}

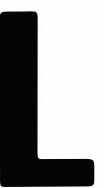

'une des premières étapes de l'action physiologique d'un messager chimique ou d'un médicament consiste en la reconnaissance de ces derniers par des organes cibles. Cette reconnaissance s'effectue par l'intermédiaire de sites de liaison spécifiques (ou récepteurs) de la substance, situés sur la membrane ou à l'intérieur de la cellule cible. La fixation de la substance sur son récepteur marque le début d'une série d'événements aboutissant à la réponse physiologique.

Au cours de ces dernières années, de nombreuses méthodologies ont été utilisées afin de démontrer l'existence de ces sites de liaison; leur principe en est simple et repose sur I'utilisation de substances radioactives marquées par différents radioisotopes (tritium, carbone-14, soufre- 35 , iode-1 25 , etc.). Ces substances peuvent ainsi émettre des rayonnements ionisants permettant de les détecter et de les mesurer. Ces rayonnements sont en outre susceptibles d'impressionner une émulsion photographique, principe de base des techniques radioautographiques. De plus, certains messagers chimiques ou médicaments peuvent être marqués par des émetteurs de positons (carbone-11, fluor-18, ou brome-75,76) ayant une demi-vie très courte (et donc sans danger) et dont la détection est rendue possible par la tomographie (technique décrite p. 424).

Ainsi, les récepteurs d'un très grand nombre de substances agissant sur le cerveau ont pu être mis en évidence, comme par exemple ceux des neuro-médiateurs (catécholamines, sérotonine, acétylcholine), des peptides (opiacés, peptides gastrointestinaux) ou de certaines hormones périphériques (stéroïdes par exemple) et de divers médicaments (neuroleptiques, anxiolytiques).

Pour cela, des approches in vivo après administration de la substance radioactive, ou in vitro sur homogénats ou coupes de tissu, ont été utilisées. In vivo, les molécules du ligand radioactif se liant sur le récepteur sont retenues plus longtemps dans le tissu que celles qui ne se lient pas. Ainsi au bout d'un certain temps, il ne reste dans le tissu étudié que des molécules liées spécifiquement au récepteur. Ces techniques in vivo posent néanmoins certains problèmes, puisqu'il est parfois difficile de détecter un nombre suffisant de particules radioactives sans mettre en danger la vie de l'individu ou de l'animal. En outre, l'existence d'une barrière hémato- 
méningée qui protège le cerveau des importantes modifications métaboliques survenant à la périphérie, empêche certaines substances comme les peptides, de parvenir aisément au niveau de leurs sites récepteurs après administration du ligand radioactif in vivo. Néanmoins, de très grands progrès ont été réalisés, en particulier grâce à des radioligands marqués par les émetteurs de positons.

Parallèlement, des techniques in vitro se sont développées, d'une part sur des préparations membranaires de différents tissus nerveux, et d'autre part sur des coupes de cerveau congelées pour une étude en microscopie optique ou non congelées pour la microscopie électronique. Ces approches in vitro permettent d'effectuer un contrôle plus précis des conditions de fixation du ligand sur son récepteur et de tester l'effet de différents agents pour une parfaite caractérisation biochimique du récepteur.

L'organisation complexe et l'hétérogénéité tissulaire du système nerveux rendent difficile la localisation précise des récepteurs des messagers chimiques dans une structure donnée du cerveau. C'est pour cette raison que des techniques radioautographiques permettant de visualiser dans le cerveau les sites de fixation de messagers chimiques et de médicaments se sont tout particulièrement développées ces dernières années.

\section{REFERENCES}

1. Young WS, Kuhar MJ. A new method for receptor autoradiography: ${ }^{3} \mathrm{H}$-opioid receptors in rat brain. Brain Res 1979; 179: 255-70.

2. Rostène W, Besson J, Broer Y, et al. Localisation par radioautographie des récepteurs des neuropeptides dans le système nerveux central. Ann Endocrinol (Paris) $1985 ; 46: 27-33$.

3. Wood PL. Multiple opiate receptors: Support for unique mu, delta and kappa sites Neuropharmacology 1982; $21: 487-97$.

4. Hornykiewicz O. Dopamine (3hydroxytyramine) and brain function. Pharmacol Rev 1966; 18 : 925-64.

5. Sadoul JL, Checler F, Kitabgi P, Rostène W, Javoy-Agid F, Vincent JP. Loss of high affinity neurotensin receptors in substantia nigra from Parkinsonian subjects. Biochem Biophys Res Commun 1984 ; 125 : 395-404.

6. Quirion R. Interactions between neurotensin and dopamine in the brain: an overview. Peptides (Fayetteville) 1983; 4: 609-15. veau de 10 à $50 \mu$ sont pratiquées à l'aide d'un cryostat ou d'un vibratome et sont incubées en présence de la substance radioactive à $+4^{\circ} \mathrm{C}$ ou à température ambiante. A la fin de la période d'incubation (temps où l'équilibre de la fixation du ligand sur son récepteur est atteint), les coupes sont lavées et certaines récupérées pour un comptage de la radioactivité. Les autres sont placées en présence d'une émulsion photographique qui sera révélée après quelques heures, jours, semaines ou mois, selon l'isotope radioactif utilisé et la quantité de radioactivité fixée sur le récepteur.

Dans les résultats décrits ci-dessous, l'impression laissée par la coupe sur un film rigide sensible aux rayonnements émis par la substance radioactive a été analysée quantitativement par un numériseur couplé à un ordinateur, et recomposée sur un écran de télévision en images en pseudo-couleurs plus facilement reconnaissables par l'œil humain. En effet, l'autoradiogramme en noir et blanc présente des zones plus ou moins sombres en fonction de l'intensité du marquage radioactif, qui vont être arbitrairement converties en une échelle de couleurs allant du bleu (région où la densité des récepteurs est la plus faible), au rouge (régions les plus denses) [2].

Localisation des sites de reconnaissance d'une drogue opiacée au niveau du système nerveux central. La figure I illustre la localisation des sites de liaison de la brémazocine tritiée, une drogue opiacée de la famille de la morphine et de l'héroïne, dans le cerveau de cobaye. La brémazocine semble agir de façon spécifique sur le site kappa, un des trois sous-types de récepteurs opiacés [3]. L'importante concentration de sites kappa dans les couches profondes du cortex pourrait rendre compte des effets secondaires sédatifs observés lors de l'administration de tels composés chez l'animal et chez l'homme. On pense que le développement d'agonistes très sélectifs de ce type de récepteur permettra peut-être d'obtenir de nouveaux médicaments très fortement analgésiques mais ne provoquant pas d'effets de tolérance et de dépendance comme c'est aussi le cas des 
morphinomimétiques actuels.

Localisation des sites de liaison

d'un neuropeptide dans le cerveau humain. La figure 2 représente la fixation in vitro d'un neuropeptide, la neurotensine iodée, au niveau de la substance noire humaine obtenue chez un individu normal (A) ou atteint de la maladie de Parkinson (B), maladie caractérisée essentiellement par la destruction des neurones dopaminergiques de la voie nigro-striatale dont les corps cellulaires sont localisés dans la substance noire [4]. On observe clairement une diminution très importante des sites de liaison de la neurotensine dans la substance noire du sujet atteint de la maladie de Parkinson [5]. On peut ainsi, grâce à cette approche radioautographique, mettre en évidence une disparition ou à l'inverse, une augmentation du nombre de récepteurs à une substance donnée dans différentes conditions physiopathologiques. On peut ainsi envisager d'orienter le traitement de certaines maladies psychiatriques non seulement en agissant directement sur les systèmes neuronaux endommagés, mais aussi en agissant sur les substances qui modulent leur activité ; dans le cas de la maladie de Parkinson, on pourrait donc penser utiliser un neuropeptide, la neurotensine, pour modifier l'activité des neurones dopaminergiques [6].

Localisation des sites de liaison en microscopie électronique. La visualisation radioautographique de sites récepteurs en microscopie électronique repose sur les mêmes principes méthodologiques que leur localisation à l'échelle tissulaire. On fait appel de préférence à des ligands radioactifs marqués à l'iode 125 , en raison de leur haute activité spécifique et de la faible énergie de leurs radiations (électrons Auger), ce qui permet d'obtenir une meilleure résolution qu'avec le tritium. Ces ligands peuvent être administrés in vivo, par voie systémique ou intracérébroventriculaire, ou in vitro, par incubation de tranches de cerveau frais ou légèrement pré-fixé (pour prévenir la dégradation de la structure fine en cours d'incubation). Bien que l'approche in vivo soit nettement plus favorable au $m / s n^{\circ} 8$ décembre 85

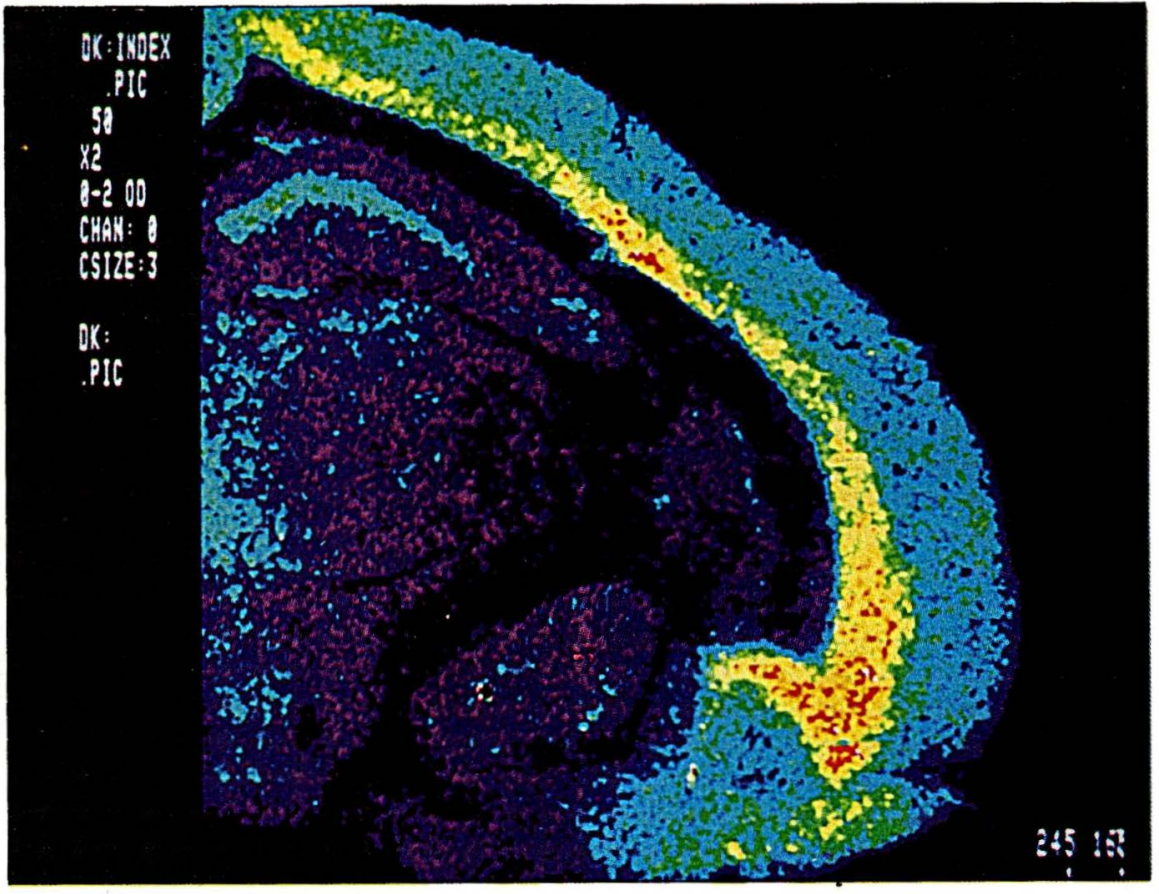

Figure 1. Fixation in vitro de la brémazocine tritiée sur les sites opiacés kappa dans le cerveau de cobaye. L'autoradiogramme a été analysé par digitalisation et transformé en une image en pseudo-couleurs où le rouge et le jaune correspondent aux régions contenant une importante concentration de récepteurs (couches profondes $d u$ cortex cérébral), alors que les zones en bleu ou violet (couches superficielles du cortex cérébral, l'hypothalamus, le thalamus et l'hippocampe) sont faiblement marquées.

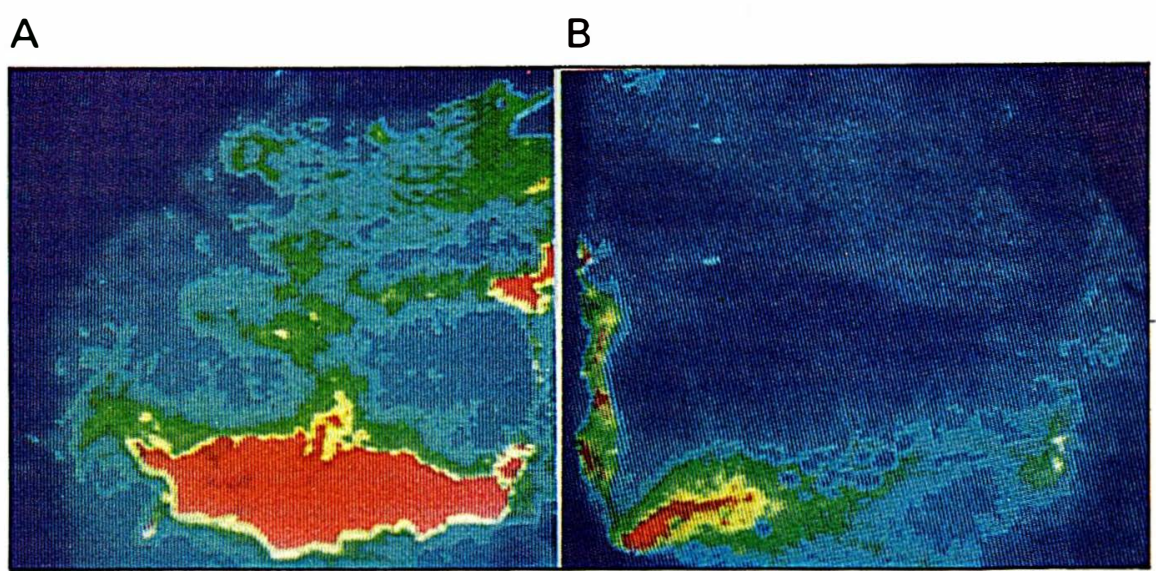

Figure 2. Fixation in vitro de la neurotensine monoiodée au niveau de la substance noire humaine chez un individu normal (A) ou chez un sujet atteint de la maladie de Parkinson (B). Les couleurs sont identiques à celles décrites dans la figure 1 . On constate la disparition presque complète des récepteurs de la neurotensine chez le sujet atteint de la maladie de Parkinson. 
plan de la préservation de la morphologie ultrastructurale, la méthode in vitro, qui permet un meilleur contrôle des conditions de liaison du traceur, s'avère souvent préférable. Dans tous les cas, on doit s'assurer que le marqueur radioactif peut être maintenu sur ses sites de liaison spécifiques au cours des divers traitements histologiques auxquels doit être soumis le tissu pour pouvoir être observé au microscope électronique. Pour prévenir la dissociation des complexes ligands-récepteurs, on a recours à des composés qui se lient de façon irréversible aux récepteurs étudiés: c'est le cas de l'alpha-bungarotoxine, un venin de serpent utilisé pour la visualisation des récepteurs cholinergiques nicotiniques de la jonction neuromusculaire [7] et de l'organe électrique du poisson [8], ou de la propylbenzilylcholine, une moutarde azotée qui se fixe de façon irréversible sur les récepteurs cholinergiques muscariniques [9]. On peut également utiliser des composés photoactivables qui, sous l'effet des rayons ultraviolets, établissent des liens covalents avec leurs sites récepteurs. Un tel ligand a permis de marquer les récepteurs aux benzodiazépines dans le système nerveux central et de démontrer qu'ils étaient proches des terminaisons nerveuses utilisant comme transmetteur l'acide gamma amino-butyrique (GABA) [10]. Une troisième approche consiste à utiliser un agent de pontage, tel la glutaraldéhyde, qui "soude", de manière covalente, le marqueur radioactif à son site récepteur. C'est le procédé que nous avons utilisé pour mettre en évidence la distribution ultrastructurale des récepteurs des opiacés [I I] et, plus récemment, de la neurotensine dans le système nerveux central.

La radioautographie illustrée sur la figure 3 provient d'une tranche de néostriatum de rat incubée en présence d'un analogue opiacé monoiodé de la met-enképhaline. On sait que dans ces conditions expérimentales, plus de $80 \%$ des molécules radioactives présentes dans le tissu sont spécifiquement liées à leurs sites récepteurs. Certaines des radiations émises par ces molécules iront impressionner l'émulsion photographique sus- jacente, provoquant le dépôt de grains d'argent. La majorité de ceux-ci chevauchent plusieurs structures nerveuses, toutes également susceptibles de contenir la source de radioactivité. Seule l'analyse statistique de la distribution d'un très grand nombre de grains d'argent permet de définir avec plus de précision la localisation des molécules de traceur. Nous sommes ainsi parvenus à déterminer que la majorité des récepteurs aux opiacés marqués par les déivés morphiniques (récepteurs de type mu) dans le néostriatum se retrouvent au niveau d'appositions membranaires. La vaste majorité des interfaces ainsi marquées sont axodendritiques $(71 \%)$, mais une proportion non négligeable $(23 \%)$ implique deux ou plusieurs terminaisons axoniques. Fait important, seul un petit nombre de sites de liaison marqués ( $7 \%$ ) paraît être associé aux différenciations membranaires qui caractérisent normalement la présence de contacts synaptiques. Cette observation suggère que les opiacées aussi bien exogènes qu'endogènes - exercent surtout leurs effets par l'intermédiaire de récepteurs non jonctionnels (extra synaptiques) dans le système nerveux central.

\section{La tomographie par émission de positons}

La tomographie par émission de positons (TEP) est une technique de visualisation non traumatisante qui permet d'obtenir, in vivo; une image quantitative de la distribution d'un radioélément émetteur de positons dans une section transverse quelconque du corps humain. C'est donc une technique qui, après administration chez l'homme d'une molécule marquée par un émetteur de positons, permet d'obtenir in vivo des résultats comparables à ceux que l'on obtient, post-mortem, à l'aide de la radioautographie quantitative.

La TEP utilise les propriétés des deux photons de $511 \mathrm{keV}$ qui résultent de l'annihilation du positon émis lors de la désintégration du radioélément. Ces photons, qui sont émis à $180^{\circ}$ l'un de l'autre, sont détectés par deux scintillateurs reliés à un circuit de coïncidence qui n'enregistre une impulsion que lorsque les deux détecteurs sont excités simultanément. En utilisant la " collimation électronique" ainsi réalisée et en multipliant les angles de détection des positons (avec, cette fois une couronne complète de détecteurs), il est possible de reconstruire par ordinateur une image quantitative, en fausses couleurs (chaque couleur correspond à un niveau d'activité), de la répartition du radioélément dans une "tranche" correspondant au plan des détecteurs. Une meilleure localisation du lieu d'annihilation du positon, le long de la ligne de coincidence électronique, est obtenue grâce à la mesure de la différence du "temps de vol " des deux photons entre le moment de leur émission et celui de leur détection par les deux scintillateurs. Les images ainsi obtenues représentent la distribution du radioélément au sein d'une tranche d'environ $10 \mathrm{~mm}$ d'épaisseur, la résolution dans cette coupe étant elle aussi d'environ $10 \mathrm{~mm}[12]$.

Cette technologie qui jusqu'à présent a surtout été utilisée chez l'homme pour des études de débit sanguin cérébral ou des études de métabolisme cérébral glucidique ou protéique est, comme nous allons le voir, une méthode tout à fait adaptée à l'étude in vivo chez l'homme des récepteurs en général et notamment des récepteurs du système nerveux central [13]. Le ligand, qui est une molécule ayant une affinité suffisante pour se fixer sélectivement sur un type de récepteurs, est "marqué " par un radioisotope émetteur de positons avant d'être administré, le plus souvent par voie intraveineuse, au sujet ou au patient. Après avoir attendu le temps nécessaire pour que la fixation spécifique soit la plus élevée possible, la cartographie de la distribution du radioisotope émetteur de positons obtenue par TEP reflète la distribution des récepteurs étudiés, à condition toutefois que le radioligand ne soit pas métabolisé. Les ligands développés pour la radioautographie quantitative ne sont pas tous utilisables en TEP mais on dispose cependant des radioligands marqués par des émetteurs de positons $\left({ }^{11} \mathrm{C},{ }^{18} \mathrm{~F},{ }^{75.76} \mathrm{Br}\right)$ nécessaires 
pour étudier les récepteurs de certains neuromédiateurs (dopamine, adrénergique, cholinergique muscarinique) et ceux des benzodiazépines et des opiacés.

Pour que les sites de fixation du radioligand ainsi mis en évidence puissent être identifiés à des récepteurs, deux critères importants doivent encore être démontrés : d'une part la saturabilité de la liaison et d'autre part la corrélation entre une variation du nombre de sites de fixation et différents états physiopathologiques. L'exemple suivant (figure 4) relatif à la visualisation in vivo des récepteurs de la dopamine dans le système nerveux central chez l'homme, illustre les possibilités de la TEP dans ce domaine. Le radioligand utilisé est un médicament neuroleptique bien connu, la spipérone, marqué par un radioisotope du brome émetteur de positons [14]. Cette molécule qui a une forte affinité pour les récepteurs de la dopamine va progressivement se concentrer au niveau des noyaux gris centraux (striatum) qui sont des

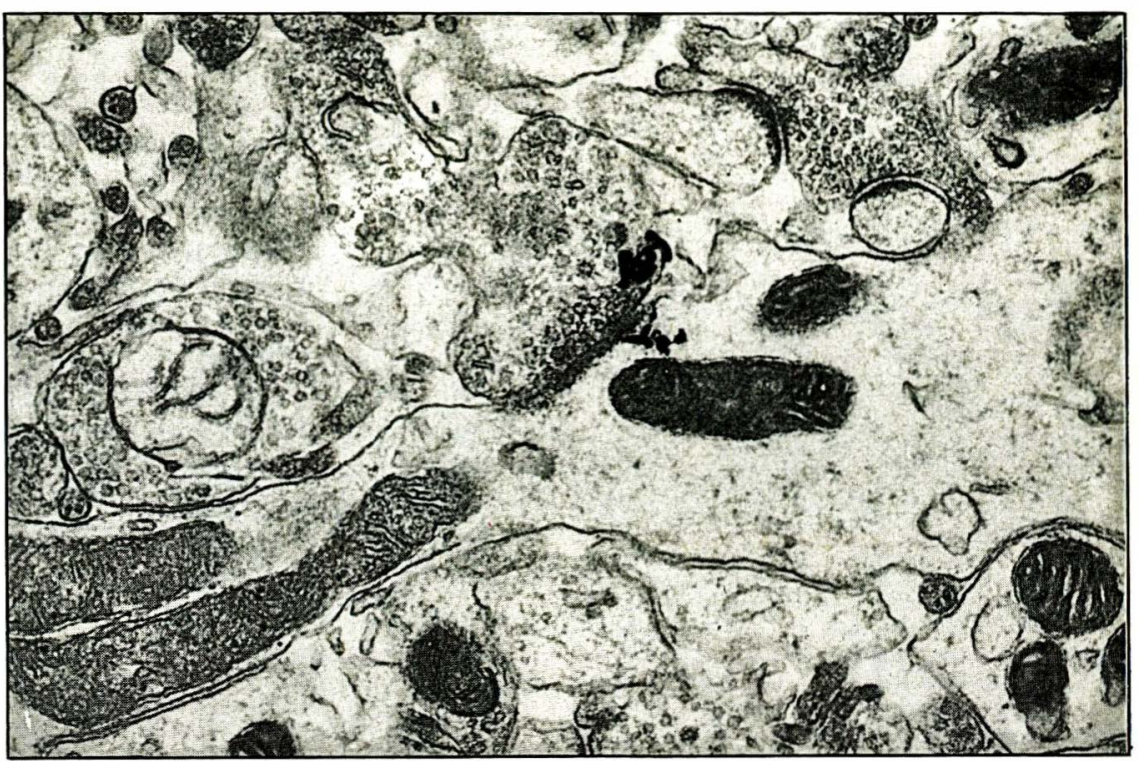

Figure 3. Radioautographie en microscopie électronique d'une coupe de néostriatum incubé en présence d'un pentapeptide opiacé monoiodé (FK 33-824, Sandoz). Les grains d'argent produits dans l'émission qui recouvre la coupe par les radiations issues des molécules radioactives fixées à leurs sites récepteurs, sont détectés sous forme de tortillons noirs. Les deux grains d'argent visibles sur ce cliché sont associés au contact synaptique établi par une terminaison axonique remplie d'organites vésiculaires sur un gros tronc dentritique $(\times 25000)$.

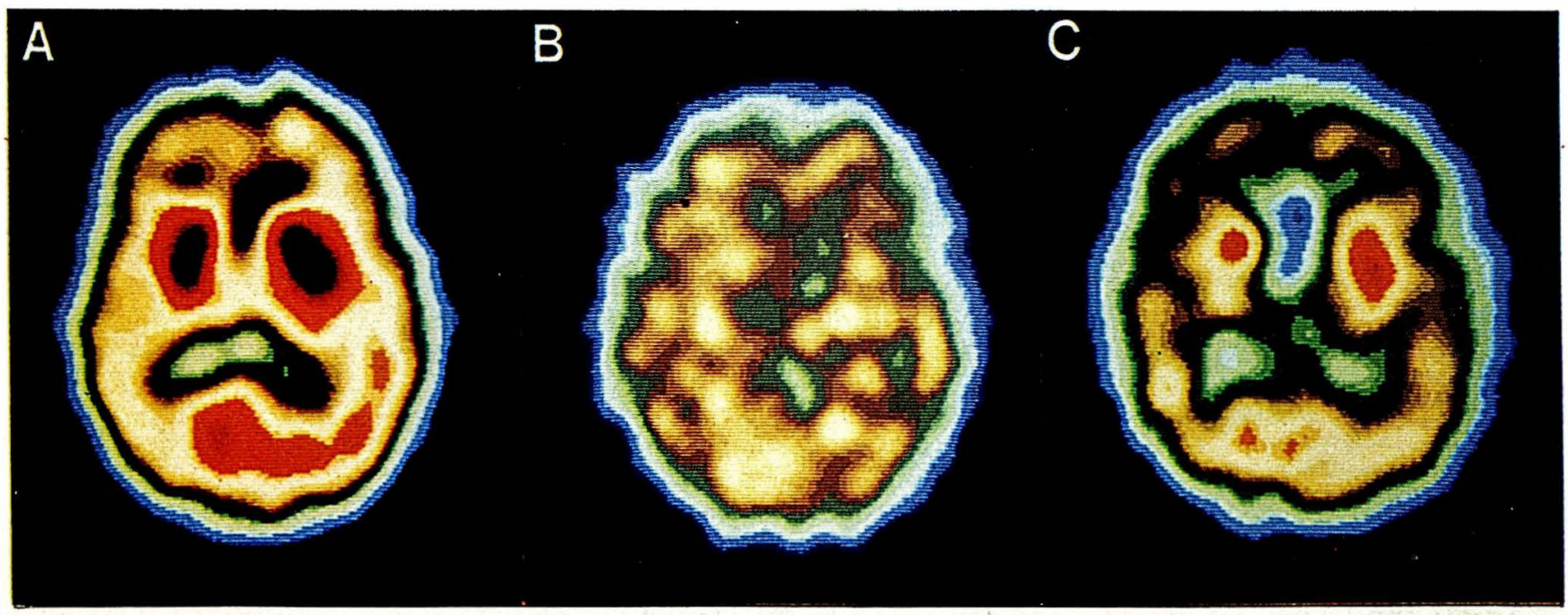

Figure 4. Images cérébrales de la localisation des sites récepteurs de la dopamine (zones apparaissant en rouge plus ou moins sombre et correspondant aux noyaux gris centraux et au cortex occipital) visualisés par tomographie d'émission de positons après injection d'un neuroleptique (spipérone) marqué par le brome-76 chez : un sujet témoin (image A), un malade traité quotidiennement avec de fortes doses de neuroleptique non radioactif (image B) et un malade souffrant d'une maladie dégénérative du système nerveux central entraînant une diminution du nombre de récepteurs de la dopamine (image $C$ ). 


\section{REFERENCES}

7. Porter CW, Barnard EA, Chiu TH. The ultrastructural localization and quantitation of cholinergic receptors at the mouse motor endplate. 3 Membr Biol 1973; 14: 383-402.

8. Bourgeois JP, Ryter A, Menez A, Fromageot $P$, Boquet $P$, Changeux JP. Localization of the cholinergic receptor protein in electrophorus electroplax by high resolution autoradiography. FEBS Lett 1972; 25: 127-33.

9. Kuhar MJ, Taylor N, Wamsley JK, Hulme EC, Birdsall NJM. Muscarinic cholinergic receptor localization in brain by electron microscopic autoradiography. Brain Res 1981 ; 216: 1-9.

10. Mohler H, Richards JG, Wu JY. Autoradiographic localization of benzodiazepine receptors in immunocytochemically identified gammaaminobutyrergic synapses. Proc Natl Acad Sci USA $1981 ; 78: 1935-8$.

11. Beaudet A, Tremeau O, Menez A, Droz B. Visualisation des récepteurs aux opiacés dans le locus coeruleus du rat : étude radioautographique à haute résolution après administration d'un analogue tritié de la met-enképhaline. $C R$ Acad $S c i$ (Paris) 1979; 289: 591-4.

12. Soussaline F, Comar D, Allemand R, Campagnolo R, Laval M, Vacher J. New developments in positron emission tomography. Instrụmentation using the time-of-flight inf ormation. In: Greitz T, éd. The metabolism of the Human Brain Studied with Positron Emission Tomography. New York: Raven Press $1985 ; 1-11$.

13. Hantraye P, Kaijima M, Prenant C, et al. Central type benzodiazepine binding sites: A positron emission tomography study in the baboon's brain. Neurosci Lett 1984; 48: 115-20.

14. Mazière $\mathrm{B}$, Loc'h $\mathrm{C}$, Hantraye $\mathrm{P}$, et al. ${ }^{76} \mathrm{Br}$ bromospiropcridol: A new tool for quantitative in vivo imaging of neuroleptic receptors. Life $S c i$ $1984 ; 35: 1349-56$.

15. Mazière $B$, Loc'h $C$, Baron $J C$, et al. In vivo quantitative imaging of dopamine receptors in human brain using positron emission tomography and ${ }^{76} \mathrm{Br}$-bromospipcrone. Eur 3 Pharmacol 1985 (sous presse).

16. Baron JC, Mazière B, Loc'h C, Sgouropoulos P, Bonnet AM, Agid Y. Progressive supranuclear palsy: Loss of striatal dopamine receptors demonstrated in vivo by positron tomography.

structures cérérales renfermant un grand nombre de ces récepteurs. Quatre heures trente après l'injection du radioligand, l'image obtenue (figure $4 A$ ) par TEP chez un sujet normal permet de visualiser et de quantifier les récepteurs de la dopamine $[15]$. L'image $B$ correspond à une image de la même coupe du cerveau obtenue chez un malade traité quotidiennement par de fortes doses d'un médicament neuroleptique semblable au radioligand, mais non radioactif. Chez ce patient, les récepteurs de la dopamine sont saturés par le médicament et le radioligand ne peut plus se lier de façon spécifique : il devient impossible de distinguer sur cette image un site de fixation quel qu'il soit. L'image $C$ correspond encore à une même coupe cérébrale obtenue, cette fois, chez un patient souffrant d'une maladie dégénérative du système nerveux central, la démence sous-corticale. On sait, grâce à des études post-mortem, que cette maladie entraîne une diminution importante du nombre de récepteurs de la dopamine au niveau du striatum. Les résultats obtenus in vivo par TEP confirment les études post-mortem : la très faible fixation du radioligand au niveau des noyaux gris centraux correspond bien à une diminution du nombre de récepteurs [16].

Il est donc possible, grâce à l'utilisation conjointe d'un ligand spécifique marqué par un émetteur de positons et de la TEP, de visualiser, de caractériser et de quantifier des sites de liaison in vivo chez l'homme.

Grâce à l'introduction de ces différentes approches méthodologiques directement liées au développement des systèmes d'analyse d'images les plus sophistiqués et aux nouvelles approches de biologie, on peut dès lors envisager l'élaboration de nouvelles stratégies permettant de sélectionner des médicaments se fixant sur une structure précise (et uniquement celle-là) et de développer de nouvelles drogues dénuées d'effets secondaires. Ceci aboutirait à accroître nos connaissances sur le fonctionnement non seulement du cerveau mais aussi d'autres organes, et à mieux comprendre l'étiologie de certaines maladies

\section{Summary}

New brain imaging technologies for the characterization and visualization of receptors for neuroactive substances allow a precise topographical distribution and the study of receptor site modifications for central chemical messengers or drugs by radioautographic methods. Binding sites may be now localized at the level of the cell itself by electronmicroscopy.

Positron emission tomography is a suitable approach for the in vivo study of those receptor sites in normal and pathological brains. Those methodologies provide new tools for the development of new drugs, and facilities for a better understanding of brain functions.

\section{TIRES A PART}

W. Rostène : unité Inserm U55, centre de recherches Saint-Antoine, 184 , rue du Faubourg-Saint-Antoine, 7557I Paris cedex 12, France. 